\title{
Erratum to: Low incidences of acute and chronic graft-versus-host disease after unrelated bone marrow transplantation with low-dose anti-T lymphocyte globulin
}

\author{
Kazuo Hatanaka Shigeo Fuji $\cdot$ Kazuhiro Ikegame $\cdot$ Ruri Kato Atsushi Wake $\cdot$ Michihiro Hidaka $\cdot$ Toshiro Ito \\ Masami Inoue $\cdot$ Yoshihisa Nagatoshi $\cdot$ Akiyoshi Takami $\cdot$ Naokuni Uike $\cdot$ Hisashi Sakamaki $\cdot$ Hiromasa Yabe $\cdot$ \\ Yasuo Morishima $\cdot$ Ritsuro Suzuki $\cdot$ Yoshiko Atsuta $\cdot$ Takahiro Fukuda
}

Published online: 25 December 2012

(c) The Japanese Society of Hematology 2012

\section{Erratum to: Int J Hematol}

DOI 10.1007/s12185-012-1209-4

A published-ahead-of-print version of this article was made available online on 7 November 2012. Subsequently an error was discovered in Fig. 4. The corrected version of

The online version of the original article can be found under doi:10.1007/s12185-012-1209-4.

\section{K. Hatanaka}

Department of Hematology/Oncology, Wakayama Medical

University Hospital, Wakayama, Japan

\section{K. Hatanaka}

Department of Internal Medicine,

Rinku General Medical Center, Izumisano, Japan

S. Fuji · T. Fukuda ( $\square)$

Hematopoietic Stem Cell Transplantation Division,

National Cancer Center Hospital, 5-1-1, Tsukiji,

Chuo-Ku, Tokyo 104-0045, Japan

e-mail: tafukuda@ncc.go.jp

\section{K. Ikegame $\cdot$ R. Kato}

Division of Hematology, Department of Internal Medicine,

Hyogo College of Medicine, Nishinomiya, Japan

K. Ikegame $\cdot$ R. Kato

Department of Medicine III,

Osaka University Hospital, Suita, Japan

\section{A. Wake}

Department of Hematology,

Toranomon Hospital, Tokyo, Japan

\section{A. Wake}

Department of Hematology,

Toranomon Hospital Kajigaya, Kawasaki, Japan
Fig. 4 is shown here. The major findings and conclusions of this paper are not affected by these changes.

M. Hidaka

Department of Internal Medicine, National Hospital

Organization, Kumamoto Medical Center, Kumamoto, Japan

T. Ito

Division of Hematology, Department of Internal Medicine,

Shinshu University School of Medicine, Nagano, Japan

M. Inoue

Department of Hematology/Oncology, Osaka Medical Center and Research Institute for Maternal and Child Health,

Iaumi, Japan

Y. Nagatoshi

Section of Pediatrics,

National Kyushu Cancer Center, Fukuoka, Japan

Y. Nagatoshi

Department of Pediatrics,

Fukuoka National Hospital, Fukuoka, Japan

A. Takami

Department of Hematology and Oncology,

Kanazawa University Hospital, Kanzawa, Japan

N. Uike

Department of Hematology,

National Kyushu Cancer Center, Fukuoka, Japan 
Fig. 4 Grade II-IV acute GVHD (a), grade III-IV acute GVHD (b), chronic GVHD (c), extensive chronic GVHD (d), overall survival (e) and non-relapse mortality (f) according to the dose of ATG-F in adult patients
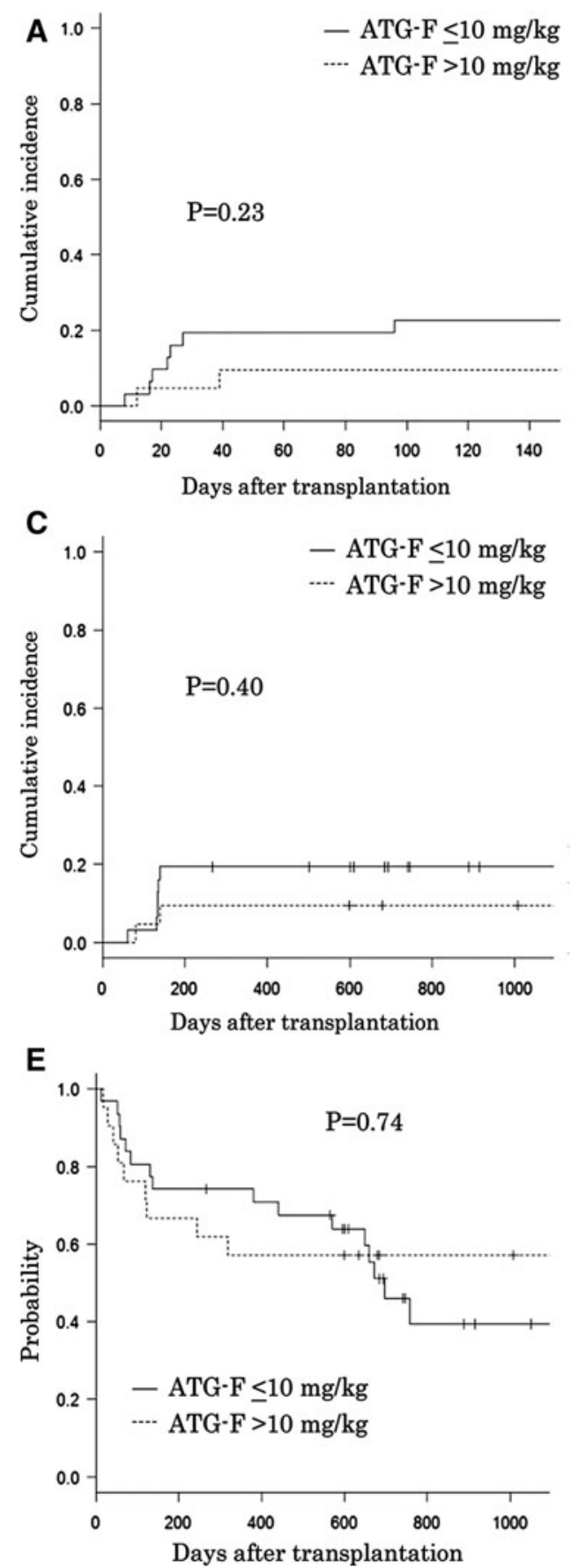

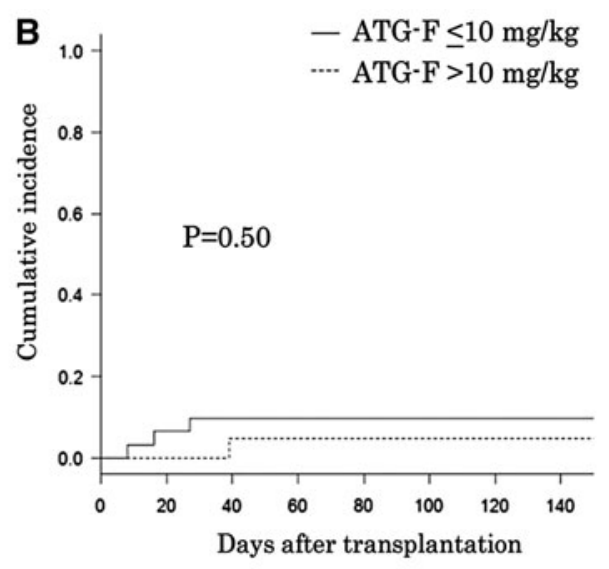

D

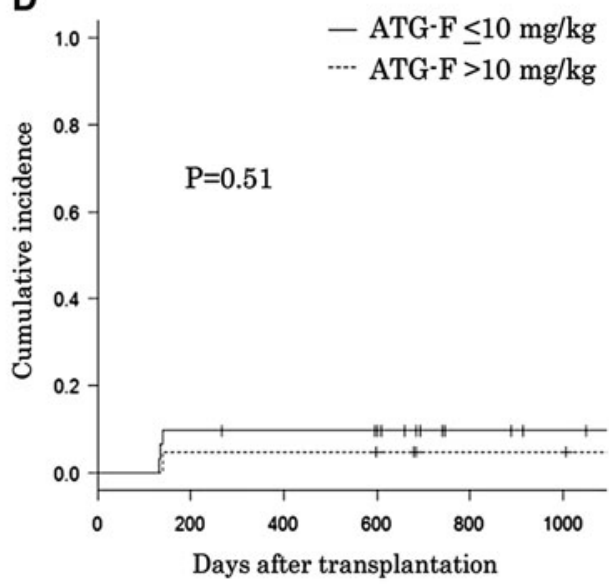

$\mathbf{F}$

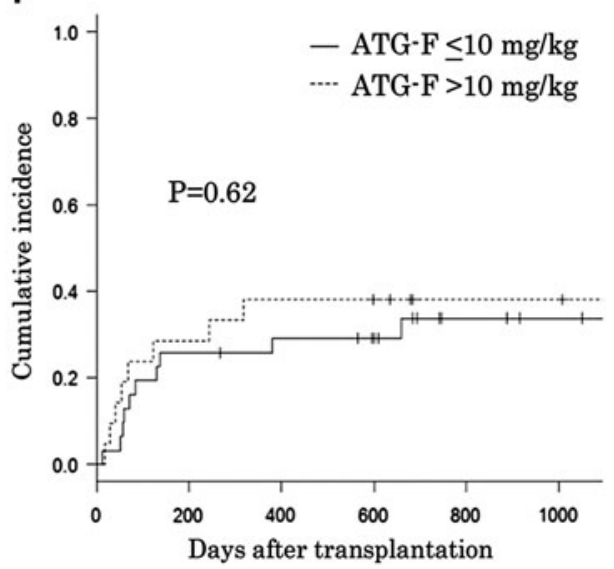

H. Sakamaki

Hematology Division, Tokyo Metropolitan Cancer and Infectious Diseases Center-Komagome Hospital, Tokyo, Japan

H. Yabe

Department of Cell Transplantation and Regenerative Medicine,

Tokai University School of Medicine, Tokyo, Japan
Y. Morishima

Division of Epidemiology and Prevention,

Aichi Cancer Center Research Institute, Nagoya, Japan

R. Suzuki - Y. Atsuta

Department of HSCT Data Management/Biostatistics,

Nagoya University, Nagoya, Japan 